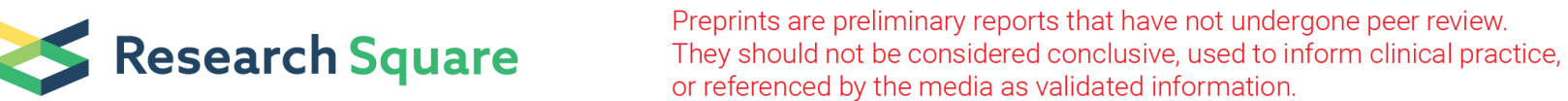

\section{NSUN2-Mediated Splice Site m5C Methylation Modification Promotes the Progression of Colon Cancer Through Modulating the Discrepant Cleavage of tRNA-Arg}

\section{$\mathrm{Na}$ Luan}

Zhejiang University https://orcid.org/0000-0002-9700-474X

\section{Yanjing Cao}

: Third People's Hospital of Hangzhou

\section{Jianguo Sun}

The Fourth Affiliated Hospital Zhejiang University School of Medicine Jiayi Mu

Zhejiang University School of Medicine Second Affiliated Hospital

\section{Yali Mu}

Zhejiang University School of Medicine Second Affiliated Hospital

\section{Xun Ye}

Zhejiang University School of Medicine Second Affiliated Hospital

Qin Zhou

Zhejiang University School of Medicine Second Affiliated Hospital

\section{Miaorong Xu}

Zhejiang University School of Medicine Second Affiliated Hospital

\section{Zhe Tang}

The Fourth Affiliated Hospital Zhejiang University School of Medicine

Jianwei Wang ( $\nabla$ sypzju@zju.edu.cn )

Zhejiang University School of Medicine Second Affiliated Hospital

\section{Research}

Keywords: Hypoxia, RNA methylation, NSUN2, m5C modification, tRNA, tRNA-derived fragments, noncoding RNA, colon cancer

Posted Date: August 31st, 2021

DOI: https://doi.org/10.21203/rs.3.rs-786758/v1 
License: (c) (i) This work is licensed under a Creative Commons Attribution 4.0 International License. Read Full License 


\section{Abstract}

Background: Hypoxia is a key driving factor for the tumour microenvironment restructuring, which leading to the variation of gene expression profiling in cancer cells. Increasing evidence reveals the initial action of hypoxia in the epitranscriptomics including RNA methylation. The role of tRNA-derived fragments (tRFs) in regulating tumour metastasis potential has attracted attention.

Methods: The expression of tRFs in colon cancer cells under hypoxia were evaluated based on fulltranscript sequencing and bioinformatics analysis and their effects on colon cancer metastasis were detected by transwell assays. The role of $\mathrm{C} 34$ was verified by introducing mutation and artificial m5C modification. The effects of NSUN2 on the biological characteristics of colon cancer cells were investigated on the basis of gain-of-function and loss-of function analyses. Lung metastasis model further uncovered the roles of NSUN2 and key tRF in tumour progression. Assays of RNA immunoprecipitation-qPCR (RIP-qPCR) were performed to identify that NSUN2 is a key methyltransferase for cysteine modification at C34 of tRNA-Arg.

Results: The present study verified the up-expression of tRF (tRF-20-MEJB5Y13) and down-expression of tRFs (tRF-20-M0NK5Y93 and tRF-21-3OPP6N7KE) in colon cancer cells under hypoxia, and all of them were derived from different tRNA-Arg. Contradictory effects of these three tRNA-Arg-derived tRFs on metastatic potential of colon cancer were demonstrated in this study. The sequence differences and the key nucleotide bases of tRNA with the methylation modification potential among the source tRNAs were analysed. Notably, our data identified C34 of tRNA-Arg as a key site that may play an important role in hypoxia-mediated tRNA-Arg discrepant cleavage. We further investigated that NSUN2 mediated specific site methylation of tRNA-Arg at C34, thereby protecting tRNA from cleavage by endonuclease and subsequently promoting the colon cancer metastasis both in vitro and in vivo.

Conclusion: The present study elaborates on the precise regulatory mechanism of m5C methylation and the role in the selective cleavage of tRNA from the perspective of noncoding RNA methylation epitranscriptomics, providing a novel insight into the molecular basis of selective expression of tRFs in colon cancer under hypoxia.

\section{Background}

Colon cancer is one of the most common global malignancies, ranking third in incidence and second in cancer-related death [1]. Colon cancer recurrence and metastasis are the main causes of treatment failure, poor prognosis and short long-term survival. Tumour metastasis is a series of complex sequential processes involving several factors, developmental steps and changes in gene regulation [2]. Alterations in gene transcription are usually caused by somatic changes in the cancer genome, and there are various forms of RNA alterations in cancer cells, including overexpression, splicing alterations, and gene fusion.

Among more than 170 known RNA modifications, methylation is the most common [3]. In RNA, 5methylcytosine ( $\mathrm{m} 5 \mathrm{C}$ ) is critical for normal development and is an important regulator of many aspects 
of gene expression, including RNA export, ribosome assembly, translation, and RNA stability. m5C modifications can occur in the acceptor stem (C72), variable loop region (C47-C50), anticodon stem (C38) and anticodon loop (C34) of transfer ribonucleic acids (tRNAs) [4]. m5C in RNA molecules is catalysed by members of the NOL1/NOP2/SUN domain (NSUN) protein family, which includes 7 members of the highly conserved human NSUN family (NSUN1-7) and the DNA methyltransferase (DNMT) homologue DNMT2 [5]. NSUN2-mediated methylation of tRNA sites occurs specifically in the anticodon or variable loop $(\mathrm{VL})$, thereby protecting tRNA from endonucleic cleavage $[6,7]$. This protection is important to prevent the accumulation of tRNA-derived fragments (tRFs), which inhibit protein synthesis. For instance, loss-of-function mutations in the human NSUN2 and NSUN3 genes lead to neurodevelopmental and mitochondrial diseases, respectively [8-11]. According to bioinformatics analysis, NSUN2, as a major m5C methyltransferase, was reported to promote cell proliferation, migration and invasion of breast cancer [12] and gallbladder cancer [13] and was relevant to the poor prognosis of patients with head and neck squamous cell carcinoma [14]. Immunohistochemical (IHC) analysis showed that the protein expression of NSUN2 was elevated in all types of cancer, including oesophageal, gastric, liver, cervical, prostate, kidney, bladder, thyroid, and breast cancers [15]. In addition to their original description of NSUN2 as an RNA guanine-7 methyltransferase (RNMT), Frye and Watt observed high expression of NSUN2 in stage III/IV colon cancer [15], which was subsequently confirmed by other researchers [16]. Intriguingly, a circular RNA (circRNA) derived from the NSUN2 coding sequence called circNSUN2 (hsa_circ_0007380) has recently been identified as being frequently upregulated in patients with colorectal cancer (CRC) and predicts poor patient survival [17]. However, the expression level of NSUN2 in colon cancer cells and its functional role in tumorigenesis and progression remain unknown.

Recent studies have revealed important biological roles of tRNA beyond gene translation. Sohailtavazoie and Hanigoodarzi, used genome sequencing technology to measure the number of tRNAs in different types of cells and confirmed that tRNAs encoding specific amino acids can promote tumour metastasis [18]. Although there are many ways to encode the same amino acid codon, it is found that only one codon of arginine corresponds to tRNA (tRNA ArgCCG, where CCG is the antisense codon of CGG) and one codon of glutamic acid tRNA (tRNA GluUUC) has the characteristics of significantly affecting tumor metastasis. By up-regulating or decreasing the expression of these two tRNAs, the metastatic potential also changes. The two codons of synonymous mutations (encoding the same amino acid) lost their transfer characteristics. It is worthy of interest that Arginine has an inseparable relationship with tumour genesis. Arginine has been identified as the only physiological precursor of NO [19]. One of the most important roles of arginine is that it participates in the synthesis of polyamines by transferring from the NO synthesis pathway. Polyamines are known to promote tumor growth, invasion and metastasis [20]. Moreover, under pathophysiological stresses such as hypoxia, some specific types of mature tRNA or precursor tRNA are specifically cleaved by endonucleases such as Dicer [21], Elac2 [22] and Ang [23] to produce tRFs or tRNA halves (tiRNAs), which belong to the short noncoding RNA family. Abnormal levels of tRFs have been observed in a number of human diseases, including cancer. Our study showed that hypoxia-induced changes in tRF levels may be closely related to the progression of colon cancer metastasis. There were significant differences in the expression of different tRFs under hypoxic 
conditions. Among the differentially expressed tRFs, two significantly downregulated tRFs (tRF-20MONK5Y93, tRF-21-30PP6N7KE) and one significantly upregulated tRF (tRF-20-MEJB5Y13) showed the most obvious expression differences, which were consistent with the trend of sequencing results (see the basis of previous work) [24,25]. All three types of tRFs are derived from tRNAs corresponding to different codons for arginine (Arg). To distinguish these tRFs in this study, we named them tRF-ArgCCG down-1, tRF-ArgCCU down-2, and tRF-ArgUCG up-1 (the database of named sources and their sequences shown in supplementary Table S1). Previous studies have confirmed that methyltransferase-mediated tRNA methylation regulates stress-induced tRNA shearing [26]. We speculated that there is some regulatory mechanism related to hypoxia-induced tRNA cleavage, and the levels of tRFs may be closely related to the specific tRNA structure and specific sites of RNA methylation. In this study, we identified C34 of tRNA as a key site that may play an important role in NSUN2 methyltransferase activity.

In mammals, the precise regulation of tRF cleavage in colon cancer under hypoxic conditions is still largely unknown. In this study, we identified an association between tRNA methylation at specific sites under hypoxic conditions (with discrepant cleavage of tRNA-Arg) and the development of conlon cancer, and explored the mechanism of NSUN2 in this process. Our findings reveal an unprecedented mechanism by which RNA with $\mathrm{m} 5 \mathrm{C}$ regulates tRNA discrepant cleavage in conlon cancer and provide potential therapeutic strategies for colon cancer.

\section{Methods}

\section{RNA sequencing}

Total RNA was extracted with TRIzol (Invitrogen, Carlsbad, CA, USA). RNA integrity was analysed with an Agilent 2100 Bioanalyzer (Agilent Technologies, Santa Clara, CA, USA). A Qubit RNA detection kit and a Qubit Fluorometer (Invitrogen, Carlsbad, USA) were used to measure the RNA concentration. The samples used in subsequent experiments met the following requirements: RNA integrity number (RIN) of tRFArgCCU down, 2.0; and 28S:18S ratio, 1.5. A sequencing library was generated and sequenced by Boao Biotechnology (Beijing, China), with $5 \mu \mathrm{g}$ of RNA per sample. Ribosomal RNA (rRNA) and linear RNA were removed from total RNA using a Ribose Zero Magnetic Kit (Epicenter Technologies, Madison, WI, USA) and RNase R (Epicenter Technologies, Madison, WI, USA), respectively. The NEBNext Ultra RNA Library Preparation Kit from Illumina (Nebraska, USA) was used to construct a sequencing library according to the manufacturer's protocol. In NEBNext first-strand synthesis reaction buffer (5x), RNA is fragmented into fragments of approximately 300 base pairs (bp) in length. First-strand cDNA was synthesized by adding reverse transcriptase and random hexamer primers to the RNA fragments, and second-strand cDNA was synthesized with 10x dUTP Mix in second strand synthesis reaction buffer. The end of the cDNA fragment undergoes an end repair process, which involves adding a single base and then connecting the Illumina sequencing adapter, and the cDNAs were digested with USER enzyme (NEB, USA) to construct a strand-specific library. The generated libraries were then purified and subjected to PCR amplification before they were certified on an Agilent 2100 Bioanalyzer system and quantified using a KAPA Library Quantification Kit. 


\section{Database analysis}

High and low expression levels of NSUN2 were defined as greater and less than the median of the values of transcripts, respectively. NSUN2 expression in colon cancer and other cancers was analysed using the Gene Expression Profiling Interactive Analysis (GEPIA) (http://gepia.cancer-pku.cn) and Human Cancer Metastasis Database (HCMDB) (http://hcmdb.i-sanger.com) online databases.

\section{Cell culture and treatments}

The human colon cacner cell lines RKO, SW480, HCT116 and CT-26 were obtained from American Type Culture Collection (ATCC, Manassas, VA, USA) and maintained in Roswell Park Memorial Institute-1640 (RPMI-1640) medium (Gibco, Life Technologies, USA) supplemented with $10 \%$ foetal bovine serum (GIBCO, USA) and 1\% penicillin/streptomycin (Invitrogen, Carlsbad, USA). Cells were routinely tested for mycoplasma contamination with a MycoAlert Mycoplasma Detection Kit. All cell lines were maintained in a constant temperature incubator with $\mathrm{CO}_{2}\left(37^{\circ} \mathrm{C}, 21 \% \mathrm{O}_{2}, 5 \% \mathrm{CO}_{2}\right)$ (Thermo Fisher Scientific, Rockford, IL, USA) for normal oxygen culture or in a hypoxic incubator with $\mathrm{CO}_{2}\left(37^{\circ} \mathrm{C}, 1 \% \mathrm{O}_{2}, 5 \% \mathrm{CO}_{2}\right)$ for hypoxic culture for $24 \mathrm{~h}$.

\section{Lentiviral vectors and transfection}

Lentiviral constructs for NSUN2 overexpression and tRF-ArgCCG down-1 knockdown were acquired from Obio Technology (Shanghai, China) and generated as previously described [27]. Prepared Colon cancer cells were stably infected with NSUN2 overexpression lentivirus (termed NSUN2) or negative control virus (termed Ctrl) using polybrene (Obio Technology, China). Similarly, cells were stably infected with negative control (termed shCtrl) and tRF-ArgCCG down-1 knockdown lentivirus (termed sh tRF-Arg ${ }^{\text {CCG }}$ down-1). Stably infected Colon cancer cells were selected for further experiments via culture in puromycin (5 $\mu \mathrm{g} / \mathrm{ml}$ ) for $1-2$ weeks.

Small interfering RNA (siRNA) oligonucleotides targeting NSUN2 were designed and synthesized by GenePharma (Shanghai China). RKO, SW480 and HCT116 cells were transfected with plasmids using Lipofectamine 2000 (Invitrogen, Thermo, Fisher Scientific, Inc.) according to the manufacturer's instructions. The NSUN2 knockdown efficiency was detected by qRT-PCR assays $24 \mathrm{~h}$ after transfection. Overexpression of NSUN2 was conducted by transfecting an expression plasmid (GV144, synthesized by GeneChem, Shanghai), and empty vector was used as the negative control. tRNA-ArgCCG down-1 inhibitor (5'-ACCCACAAUCCCCAGCUCCG-3') and negative control (NC-inhibitor, 5'UUCUCCGAACGAGUCACGUTT-3') were purchased from GenePharma (Shanghai, China). Cells were cultured for $24 \mathrm{~h}$ after transfection prior to use in experiments.

\section{Cell migration and invasion assay}

The migration and invasion of Colon cancer cells were evaluated by Transwell assays, which were conducted with CytoSelect ${ }^{\mathrm{TM}}$ 24-well Cell Invasion assay kits. Briefly, polycarbonate filters (8- $\mu \mathrm{m}$ pore size, Corning) with or without 50\% Matrigel (BD Bioscience, Bedford, MA) coating in the upper chamber were 
inserted into the wells of a 24-well plate for assessment of migration and invasion, respectively. Note that Matrigel was not required for the migration assay. Then, after cell transfection for $48 \mathrm{~h}$, cell suspensions were seeded into the upper chamber, while $500 \mu \mathrm{l}$ of complete Dulbecco's modified Eagle's medium was placed into the lower chamber. After $24 \mathrm{~h}$ of culture at $37^{\circ} \mathrm{C}$, the cells were fixed in methanol for 20 minutes, washed with phosphate-buffered saline (PBS) 3 times, and stained with $2 \%$ crystal violet solution. Images of migrated and invaded cells in each well were captured, and the number of cells in 35 randomly selected fields under a microscope was counted. Each assay was conducted in triplicate.

\section{RIP-qPCR}

The RIP assay was performed as described previously [28] with a Magna RIP RNA-Binding Protein Immunoprecipitation Kit (Millipore, USA). Briefly, RKO and SW480 cells were lysed with RIP lysis buffer before $A / G$ magnetic beads were used to immunoprecipitate antibodies that targeted RNA-binding proteins (RBPs). The magnetic bead binding complex was fixed with a magnetic frame, and unbound material was washed away. RNA was extracted with TRIzol (Invitrogen) and subjected to qRT-PCR analysis.

\section{RNA isolation and quantitative real-time PCR (qRT-PCR)}

Total RNA was prepared from cell lines and tissues using TRIzol reagent (Thermo Fisher Scientific) according to the manufacturer's instructions. Then, total RNA was treated with TURBO DNase (AM2239; Invitrogen) for 30 minutes for further purification following the manufacturer's instructions. The RNA in each sample was washed with ethanol before its concentration and purity were assessed using a NanoDrop ND2000 (Thermo Scientific Inc., Waltham, MA) following the kit's instructions. Reverse transcription was performed using a cDNA Synthesis Kit (TaKaRa, Beijing, China).

qRT-PCR for each sample comprised $1 \mu \mathrm{l}$ of cDNA, $3.5 \mu \mathrm{l}$ of RNase-free water, $0.5 \mu \mathrm{l}$ of a predesigned Nsun2 probe (Hs00214829_m1; Applied Biosystems) and $5 \mu \mathrm{l}$ of TaqMan Fast Universal PCR Master Mix (4366073). qRT-PCR and data acquisition were performed with a Real-Time PCR System (Applied Biosystems).

The tRFs were reverse transcribed using Moloney murine leukaemia virus (M-MLV) reverse transcriptase (Promega, Madison, WI, USA). The forward and reverse primers of tRFs were designed and synthesized (Supplementary Table S2). qRT-PCR amplifications were performed using SYBR Green qPCR Master Mix $(A B I, 4367659)$ on an $A B I 7300$ real-time PCR detection system (the main primers used in this study are shown in Table S1).

\section{Western blotting assay}

Proteins were collected from cell lines following the manufacturer's protocol (KeyGEN BioTech). The protein concentration of each sample was assessed using the Pierce BCA Protein Assay kit (23225; Thermo Fisher) according to the manufacturer's instructions and a spectrophotometer with SoftMax software. Total protein from lysates was separated using sodium dodecyl sulfate-polyacrylamide gel vertical electrophoresis and then transferred onto polyvinylidene fluoride membranes (PVDF, Millipore, 
USA). The membranes were immersed with specific primary antibodies and then probed with secondary antibodies after they were washed three times with $0.1 \%$ Tris-HCl with Tween-20 (TBST). The primary antibodies used in this study were as follows: anti-MMP-2 (Abcam, ab92536, 1:1000), anti-MMP-7 (CST, 71031, 1:1000), anti-MMP-3 (CST, 14351, 1:1000), anti-MMP-9 (CST, 13667, 1:1000), anti-ZEB1 (CST, 3396, 1:1000), anti-NSUN2 (Proteintech, 66580-1-IG, 1:1000), anti-N-cadherin (CST, 13116, 1:1000), anti-Ecadherin (CST, 3195, 1:1000), anti-Vimentin (CST, 5741, 1:1000), anti-Slug (CST, 9585, 1:1000), anti-Snail (CST, 3879, 1:1000), anti-Claudin-1 (CST, 13255, 1:1000), anti-C-myc (CST, 5605, 1:1000), anti-TIMP1 (CST, 8946, 1:1000), and anti-TIMP2 (CST, 5738, 1:1000).

\section{In vivo studies}

Animal studies were performed under the experimental animal use guidelines of the National Institutes of Health and Use Committee of Zhejiang University, China. Male athymic BALBC white mice aged 5 weeks were used. For the in vivo lung metastasis model, mice were injected with CT-26 cells transduced with pcDNA3.1/NC mimics, NSUN2+/NC mimics, pcDNA3.1/tRNA-ArgCCG down-1 mimics, or NSUN2+/tRNAArgCCG down- 1 mimics ( $5 \times 10^{6}$ cells per mouse, $n=8$ for each group) diluted in $400 \mu$ l of a PBS/Matrigel (BD Biosciences) mixture (1:1). Eight weeks after injection, mice were killed, and metastatic lung tumours were analysed. The largest tumor was taken from the mouse lung and its volume was measured. The tumour volume was calculated using the formula $V=1 / 2 \times$ larger diameter $\times(\text { smaller diameter })^{2}$. Lung metastasis models, and tail vein injection models were performed. Metastases were detected using the IVIS Lumina II system (Caliper Life Sciences, Hopkinton, MA) for 10 minutes to observe GFP fluorescence in the lung.

\section{Statistics}

Each assay was independently performed at least 3 times. Statistical analyses were performed using GraphPad Prism 8.0.3 software. Student's t-test (two tailed) and ANOVA were used to detect differences between two groups or among more than two groups, respectively. The Western blot results were quantified by ImageJ software (National Institutes of Health). All data are shown as the means \pm standard deviation (SD).

\section{Results}

\section{Identification of significantly changed tRFs and their roles in Colon cancer metastasis under hypoxia}

In this study, we observed that the expression of 18 tRFs differed significantly in colon cancer cells under hypoxic conditions (Fig. 1a-c). Among them, three tRFs showed the most significant difference, with two showing decreased expression decreased and one showing increased expression. Interestingly, all three were derived from tRNAs corresponding to different codons encoding Arg; the tRFs were labelled as tRFArgCCG down-1, tRF-ArgCCU down-2 and tRF-ArgUCG up-1. Therefore, tRF mimics and tRF inhibitors were transfected into RKO, SW480 and HCT116 cells. The transfection efficiency was detected by PCR assay, 
and then the the effect of key tRFs on DNA synthesis activities were detected by flow cytometry and the analysis showed no remarkable differences among three groups (Additional file 1: Figure S1g, $\mathrm{h}$ ). Migration abilities were observed by transwell assays. The transwell assays results showed that downregulation of tRF-ArgCCG down-1 and tRF-ArgCCU down-2 could be associated with advanced progression of colon cancer cells, whereas upregulation of tRF-ArgUCG up-1 could drives colon cancer cells metastasis in vitro (Fig. $1 \mathrm{~d}-\mathrm{k}$ ). ${ }^{* *} \mathrm{P}<0.01$. The assays were repeated more than three times.

\section{tRNA Arg C34 methylation exerted critical role in cleavage and colon cancer metastasis}

By comparing the sequences of the three tRFs, we found base differences at C34. Notably, C34 happens to be the first base of the antisense codon and is a potential site for $\mathrm{m} 5 \mathrm{C}$ modification. More importantly, the location of the differential tRF on the corresponding tRNA and C34 are as follows. C34 of tRF-ArgUCG up-1 corresponding to tRNA-Arg is uracil $(U)$ rather than $C$, while the same position on tRF-ArgCCG down-1 and tRF-ArgCCU down-2 corresponding to tRNA-Arg is C (Fig. 2a). To verify that C34 is indeed the tRNA methylation modification site and is the root cause of the differential expression of tRF-ArgCCG down-1, tRF-ArgUCG up- 1 and tRF-ArgCCU down-2 under hypoxic conditions, we mutated the $C$ at position 34 to $U$ in tRNA-ArgCCG down-1 and tRNA-ArgCCU down-2 and the $U$ at position 34 to $C$ in tRNA-ArgUCG up-1.

These constructs were transfected into cells, and the levels of tRF-ArgCCG down-1 and tRF-ArgCCU down2 increased while those of tRF-ArgUCG up-1 decreased (Fig. 2b-d). Subsequently, we artificially methylated position 34 of tRNA-ArgCCG down-1 and tRNA-ArgCCU down-2, and the methylated efficiency was detected by mass spectrum (Additional file 2: Figure S2a, b) and the qPCR assays results showed that the expression of tRF-ArgCCG down-1 and tRF-ArgCCU down-2 decreased (Fig. 2e-g).

To determine the key role of C34 demethylation of tRNA-Arg, we investigated C34-methylated colon cancer cells. Wild-type and C34-methylated tRF-ArgCCU down-1 and tRF-ArgCCU down-2 constructs and the blank control were transfected into RKO, SW480, and HCT116 cells. The effects of the wild-type, C34 methylation of tRNA ArgCCU down-1 and tRF-ArgCCU down-2 on the proliferation of RKO, SW480 and HCT116 cells were examined by MTT assay. The results showed that RNA ArgCCU down- 1 and tRFArgCCU down-2 could inhibit colon cancer cells proliferation. However, after tRNA Arg C34 methylation, the inhibition lessened (Additional file 2: Figure S2c, d, e). Interestingly, the results showed that compared to the NC groups, the tRNA-ArgCCG down-1 and tRNA-ArgCCU down-2 groups could obviously inhibit colon cancer cell invasion and migration. However, transfection with mutant tRNA-ArgCCG down-1 or tRNA-ArgCCU down-2 reduced the inhibition of colon cancer cell invasion and migration compared to that of cells without m5C methylation at C34 (Fig. 2h-s). ${ }^{\star} P<0.05,{ }^{*} \mathrm{P}<0.01 .{ }^{* \star *} \mathrm{P}<0.001$. The assays were repeated more than three times.

\section{NSUN2 overexpression distinguished colon cancer with predictive values}

Sequencing results showed that there were 16 kinds of $\mathrm{m} 5 \mathrm{C}$ methylation-related enzymes and others associated with RNA cleavage, which expressed significantly different compared to normoxia group (Fig. 
3a, b). qRT-PCR results and Western Blot were performed to verify the sequencing results and all showed that the expression of NSUN2 is significantly increased under hypoxic conditions (Fig. 3c, d, e).

Comparisons between metastatic and nonmetastatic foci in the database were performed to screen out differences in NSUN2 expression and the potential correlation between NSUN2 and colon cancer. NSUN2 expression in primary colon cancer tumours is obviously higher than that in the primary normal tissue

(Fig. 3f, g). Notably, NSUN2 expression in primary tumours that metastasized was much higher than that in tumours without metastasis (Fig. 3h). These data indicate that NSUN2 may contribute to the progression of colon cancer. We also analysed the expression of other NSUN methyltransferases (NSUN 3-7) but failed to obtain positive results (Fig. 3i-r). This also verified the conclusion we made in our previous work that NSUN2 maight play a key role in modulating progression of colon cancer. Thus, we mainly focused on NSUN2 in this study. ${ }^{*}<0.05$. The assays were repeated more than three times.

\section{NSUN2 served as a promoter of colon cancer}

RKO and SW480 cells were treated with a short hairpin RNA specific for NSUN2, resulting in approximately $90 \%$ reduction of its level when compared to that of PLK0.1 group (Figure Sa, b). Further, after the NSUN2 overexpression plasmid was transfected into colon cancer cells, the expression of NSUN2 mRNA and protein levels in the cells were significantly increased (Figure S3c, d). PCR assay results showed that upon NSUN2 overexpression, the levels of tRF-ArgCCG down-1 and tRF-ArgCCU down2 decreased while those of tRF-ArgUCG up-1 remained basically unchanged (Fig. 4a-f). In contrast, after NSUN2 knockdown, the expression of tRF-ArgCCG down-1 and tRF-ArgCCU down-2 increased, while the expression of tRF-ArgUCG up-1 remained basically unchanged (Fig. 4i-n). Interestingly, transwell assays analysis showed that overexpression of NSUN2 could increase the invasion and migration ability, while down-regulation of NSUN2 could inhibit the invasion and migration ability of colon cancer cells (Fig. $4 \mathrm{~g}$, $h, 0, p)$. NSUN2 overexpression led to a decreased cell proliferation rate in the NSUN2 overexpression group compared to that of the pcDNA 3.1/vector group (Figure S3e, f). A flow cytometry assay further demonstrated G2/M arrest were observed after NSUN2 overexpression (Figure S3g, h). ns $>0.05$, *P< $0.05,{ }^{*} \mathrm{P}<0.01$. The assays were repeated more than three times.

\section{NSUN2 exerts effects on the EMT and metastasis-related markers of colon cancer}

Based on the fact that the epithelial-mesenchymal transition (EMT) is one of the dominating procedure and mechanisms for tumor metastasis, we further evaluated the effects of NSUN2 on EMT-related markers to further verify the ability of NSUN2 to promote the invasion and migration of colon cancer cells. Western blot analysis showed that NSUN2 overexpression could increase the expression of mesenchymal markers (such as N-cadherin, and Vimentin), the EMT-related transcription factors (ZEB1, Slug, Snail), invasion-related enzymes (such as MMP2, MMP3, MMP7, MMP9) and decrease the expression of epithelial markers (E-cadherin) (Fig. 5a, b, n, o). QPCR assays were performed to verify the changed mRNA expression of EMT-related markers after NSUN2 overexpressed plasmids transfection in 
RKO and SW480 cells (Fig. 5c-m, 5p-z). These results indicated that NSUN2 could influence EMT process of colon cancer cells to modulate colon cancer progression. ${ }^{\star} P<0.05,{ }^{\star} * P<0.01$. ${ }^{* \star} P<0.001$. The assays were repeated more than three times.

\section{The interaction between NSUN2 and methylated C34 of tRNA-Arg determined tRNA-Arg cleavage}

RIP-qPCR was applied to detect the changes in the binding between NSUN2 and tRNA-ArgCCU down-1, tRNA-ArgCCU down-2 after using NSUN2 with flag. As expected, when compared to the input group, NSUN2 overexpression markedly increased the binding between NSUN2 and both tRNA-ArgCCG down-1 and tRNA-ArgCCU down-2. Thus, the analysis of qPCR results showed obvious elevated expression of tRNA-ArgCCG down-1 and tRNA-ArgCCU down-2 after NSUN2 pulldown (Fig. 5a, b). Furthermore, we used an $\mathrm{m} 5 \mathrm{C}$ antibody to pull down tRNA-Arg with m5C methylation. The RIP-qPCR results showed that when NSUN2 was overexpressed, tRNA-ArgCCG down-1 and tRNA-ArgCCU down-2 were enriched upon pulldown with an m5C-specific antibody.

To further elucidate whether position 34 of tRNA-Arg determines its enzymatic cleavage and whether NSUN2 is a key methyltransferase for cysteine modification at position 34 of tRNA, we transfected tRNAArgCCG down-1 and tRNA-ArgCCU down-2 with mutations at position 34 into colon cancer cells and detected the levels of tRF-ArgCCG down-1 and tRF-ArgCCU down-2, respectively, after overexpression of NSUN2. Analysis revealed that consistent with our previous results, the levels of tRF-Arg CCG down-1 and tRF-ArgCCU down-2 in cells transfected with mutant tRNA were markedly lower than those in cells transfected with wild-type tRNAs after over-expression of NSUN2. In cells transfected with wild-type tRNAArgCCG down-1 and tRNA-ArgCCU down-2, overexpression of NSUN2 led to reductions in the levels of tRFArgCCG down-1 and tRF-ArgCCU down-2. However, in cells transfected with position 34 mutants of tRNAArgCCG down-1 and tRNA-ArgCCU down-2, NSUN2 overexpression hardly played a role in lowering the levels of tRF-ArgCCG down-1 and tRF-ArgCCU down-2 (Fig. 6f, h, j, I). Thus, the results demonstrate that NSUN2 is a key methyltransferase that functions by methylating cysteine at position 34 of tRNA, which, under hypoxic conditions, protects tRNA from cleavage. ${ }^{*} \mathrm{P}<0.01$. ${ }^{*} * \mathrm{P}<0.001$. The assays were repeated more than three times.

\section{NSUN2 promotes colon cancer metastasis partly via tRNA- ArgCCG down-1 in vitro and in vivo}

To further verify the relationship between the m5C methyltransferase activity of NSUN2 and tRNA-ArgCCG down-1, we used lentivirus to stably overexpress NSUN2 and tRNA-ArgCCG down-1 either separately or simultaneously in cells. It was found that overexpression of tRNA-ArgCCG down-1 can partially eliminate the effect of NSUN2 on suppressing tRNA-ArgCCG down-1 expression (Fig. 7a, c). More interestingly, tRNA-ArgCCG down-1 had a negative feedback effect on NSUN2 expression (Fig. 7b, d). To further illuminate the influence of both NSUN2 and tRNA ArgCCG down-1 overexpression on the migratory and invasive activity of colon cancer cells, we performed transwell assays and found that up-regulation of tRNA ArgCCG down-1 could significantly break role of promoting metastasis of NSUN2 in vitro (Fig. 7e, f). 
To investigate the effect of tRNA-ArgCCG down-1 on tumour formation and metastasis in vivo both independently and concomitantly with NSUN2 overexpression, CT-26 cells were implanted subcutaneously into the right flank of BALBC white mice as an orthotopic PC model or intravenously into the tail vein to observe lung metastasis. The BALBC white mice were divided into four groups, pcDNA 3.1/ NC mimics, NSUN2+/ NC mimics, pcDNA 3.1/ tRNA ArgCCG down-1 mimics, NSUN2+/ tRNA ArgCCG down-1 mimics, respectively.

Furthermore, we developed a lung metastasis model (via tail vein injection) to highlight the effect of NSUN2 and tRNA-ArgCCG down-1 and used an in vivo optical imaging system (IVIS) for small mice. The most typical picture is showed as followed. BALBC white mice implanted with NSUN2+/NC CT-26 cells exhibited significantly higher GFP activity in the lung than those implanted with NC cells. In contrast, the GFP signal of mice bearing pcDNA 3.1/tRNA-ArgCCG down-1 mimic RKO cells was much lower than that of mice bearing NC cells. Notably, the GFP signal of mice in the NSUN2+/tRF-ArgCCG down-1 mimics group was similar to that of the NC group (Fig. 7g), which indicated that tRF-Arg CCG down-1 could inhibit tumour metastasis in vivo and weaken the role of NSUN2 promoting metastasis. Then we removed the lung tissues of the mice, stained it and took pictures, and got similar results (Fig. 7h). The largest tumor was taken from the mouse lung and its volume was measured. The tumour volume of each group is V1 = $0.259 \mathrm{~mm}^{2}, \mathrm{~V} 2=0.668 \mathrm{~mm}^{2}, \mathrm{~V} 3=0.118 \mathrm{~mm}^{2}, \mathrm{~V} 4=0.263 \mathrm{~mm}^{2}$, respectively. ${ }^{*} \mathrm{P}<0.05, * \star \mathrm{P}<0.01 . * \star * \mathrm{P}<$ 0.001 . The assays were repeated more than three times.

\section{Schematic diagram of overall study}

Under hypoxic stress conditions, tRNA is cleaved into tRFs, some of which show increased levels (tRF-Arg UCG up-1) and others that show decreased levels (tRF-Arg CCG down-1, tRF-Arg CCU down-2). The methylation of $\mathrm{C} 34$ on the source tRNA point and the level of methylation were analysed. Furthermore, the role of the key m5C methyltransferase NSUN2, which regulates the delicate balance of endonucleasemediated tRNA cleavage, in the changes in tRF levels was clarified, and the effects of selective tRNA cleavage on metastatic potential were verified (Fig. 8).

\section{Discussion}

tRFs can regulate a variety of biological processes [29]. Aberrant expression of tRFs have been observed in a variety of human diseases, including cancer, neurodegenerative diseases, and acquired metabolic diseases [29-31]. In this study, we found that the antisense codons of tRNA-Arg encoding different codons of Arg protected tRNA-Arg via m5C methylation at their respective bases at $\mathrm{C} 34$, thus reducing the sensitivity to of these tRNAs to enzyme digestion under stress conditions. Here, we observed a trend of increased NSUN2 expression in colon cancer cells under hypoxic conditions and determined that the upregulation of NSUN2 expression was significantly associated with tumour metastasis. We demonstrated that NSUN2 is the key methyltransferase responsible for the m5C modification at C34 of tRNA-Arg in colon cancer and is a prognostic factor for colon cancer. In addition, we found that NSUN2 promoted colon cancer migration and invasion in vitro and in vivo. Consistent with this, clinical data 
showed that there were higher levels of NSUN2 in tumour tissues than in adjacent normal tissues and that these increases were associated with poorer outcomes in patients.

M5C is an abundant RNA modification that is present in a wide variety of RNA classes, including cytoplasmic and mitochondrial ribosomal RNAs (rRNAs) and tRNAs, as well as messenger RNAs (mRNAs), enhancer RNAs (eRNAs) and a number of noncoding RNAs. The m5C modification is involved in many mammalian processes, such as RNA cleavage, protein translation, and stem cell renewal [32]. In eukaryotes, C5-methylation of Cs in RNA is catalysed by enzymes of the NOL1/NOP2/SUN domain (NSUN) family and by DNMT2. The first methylated substrate of NSUN2 was identified as tRNA [4]. Previous studies identified three direct tRNA targets of human NSUN2: tRNA-Leu (C34), tRNA-Asp (C48, 49) and tRNA-Gly $(C 48,49,50)[33-35]$. Brzezicha et al. showed that NSUN2 mediated methylation in an intron-dependent manner at C34 of pre-tRNA-Leu in HeLa cells [34]. Here, upon comparing the sequences of the three tRFs with changes in their levels, we discovered different bases at C34. Notably, position 34 is the first base of the antisense codon, which is a potential site for m5C modification. More importantly, position 34 of tRF-ArgUCG up-1 corresponding to tRNA-Arg is $U$ rather than $\mathrm{C}$, while same position of tRNA ArgCCG down-1 and tRF-ArgCCU down-2 corresponding to tRNA-Arg is $\mathrm{C}$. The results suggest that the tRNA-Arg antisense codons encoding Arg protect C34 of tRNA-Arg via NSUN2-mediated m5C methylation in colon cancer cells.

NSUN2 is involved in a variety of biological pathways [15], and overexpression of NSUN2 has been found in a variety of human cancer types [16]. In recent years, many studies have revealed the abnormal expression of NSUN2 in cancer and the role it plays in the pathogenesis and development of cancer [36, 37]. Tatsuka et al. demonstrated that NSUN2 expression was abnormally upregulated in breast cancer [16]. A previous study also found that DNA hypomethylation is associated with increased NSUN2 expression in breast cancer. Overexpression of NSUN2 promotes cell proliferation, migration and invasion, while knocking out NSUN2 inhibits these processes in vivo and in vitro (overexpression of NSUN2 due to DNA hypomethylation is associated with metastatic progression in human breast cancer). In skin cancer, when the expression levels of NSUN2 were compared in normal skin with early and advanced skin cancers, loss of NSUN2 expression was associated with increased malignancy of the tumour and with an increase in the number of tumour-initiating cells [38].

High expression of NSUN2 was observed in patients with stage III/IV colon cancer [15], and this observation was subsequently confirmed by other studies [16]. However, few studies have focused on the role and underlying mechanism of NSUN2 in the development of colon cancer. Interestingly, a circRNA derived from the NSUN2 coding sequence named circNSUN2 (hsa_circ_0007380) was recently found to be upregulated in patients with colon cancer and predicted a poor prognosis [17].In models of patientderived xenotransplantation (PDX), circNSUN2 appears to promote liver metastasis when overexpressed. In addition, the circNSUN2/IGF2BP2 complex can lead to the promotion of EMT in colon cancer cells when bound to HMGA2 mRNA [17]. In the present study, we found that NSUN2 expression was upregulated in colon cancer, which could result in aberrant m5C modification at a specific site (C34) of 
tRNA-Arg. Furthermore, increased NSUN2 expression was significantly associated with colon cancer progression in vivo or in vitro and indicated a poor prognosis.

Fundamentally, we observed that there were significant differences in the expression of tRFs. Among them, the expression of three tRFs showed the most significant differences, with two tRFs showing decreased expression and one showing increased expression, but all three were derived from tRNAs corresponding to codons that encoding Arg. This suggests that tRNAs encoding different codons of the same amino acid (e.g, Arg) are differently cleavage and produce different tRF subsets. Through the indepth study, we finally confirmed that NSUN2-mediated splice site m5C methylation by acting on C34, leading to discrepant cleavage outcomes of tRFs. Our previous study confirmed that tRNA-ArgCCG down1 significantly affects the EMT process of colon cancer cells by regulating the expression of Claudin-1 [24]. In this study, the differential expression of these tRF subsets showing contradictory effects on the metastatic potential of colon cancer. In the present study, we confirmed that NSUN2 was the key methyltransferase in the methylation of the $\mathrm{C}$ at $\mathrm{C} 34$ in tRNA and this modification could reduce the cleavage efficiency of enzymes and protect tRNA from cleavage under hypoxic stress conditions. In the present study, we first showed that NSUN2 expression was significantly upregulated in colon cancer cells

under hypoxic conditions. Moreover, NSUN2 can promote the metastasis of colon cancer cells in vitro and in vivo. Further studies revealed that specific tRFs are potential downstream molecules of NSUN2 regulation in colon cancer. In addition, NSUN2 can promote the metastasis of colon cancer cells in an m5C-dependent manner. This study suggests that NSUN2-mediated m5C methylation of tRFs is a novel mechanism involved in the development of colon cancer.

\section{Conclusions}

The present study on the methylation of noncoding RNA and its effect on abberant transcriptome levels further elaborates the selectivity of m5C tRNA modifications on regulating the shearing process and finely adjust the mechanism involved. Research on tRFs which derived from tRNA-Arg under hypoxia revealed significant differences in their expression at the molecular level, revealed the site differences among these three kinds of tRNA C34 and verified that the expression of NSUN2 was up-regulated in colon cancer cells. Significantly, NSUN2-mediated splice site m5C methylation led to different cleavage outcomes of tRFs by acting on C34, playing a key role in promoting colon cancer metastasis. Our study focuses on changes in the tumour epigenetics transcriptomics with respect to tumour metastasis potential, and provided a deeper understanding of tumour metastasis in the molecular regulatory mechanism of initiating.

\section{Abbreviations}

EMT epithelial-to-mesenchymal

CRC colorectal cancer 
m5C 5-Methylcytosine

tRFs tRNA-derived fragments

ncRNA non-coding RNA

qRT-PCR quantitative real-time polymerase chain reaction

MTT 3-(4,5)-dimethylthiahiazo(-z-yl)-3,5-diphenytetrazoliumromide

NC negative control

SDS-PAGE sodium dodecyl polyacrylamide gel electrophoresis

PVDF polyvinylidene fluoride

\section{Declarations}

\section{Ethics approval and consent to participate}

The research protocol was reviewed and approved by the Ethical Committee of The Second Affiliated Hospital of Zhejiang University School of Medicine.

\section{Consent for publication}

Not applicable.

\section{Availability of data and material}

The datasets used and/or analysed during the current study are available from the corresponding author on reasonable request.

\section{Competing interests}

The authors declare no conflict of interests regarding the publication of this paper.

\section{Funding}

This study was supported by the National Natural Science Foundation of China (No. 81672364, No. 81871917 and No.81802988).

\section{Authors' contributions}

JW and ZT provided the idea of the study. NL, YC, and JS conceived and designed the experiments. NL, $\mathrm{JM}, \mathrm{YM}, \mathrm{XY}, \mathrm{QZ}$ and MX performed the experiments. NL, JW and ZT analyzed the bioinformatics data. NL 
wrote the draft. YC, JS, ZT, and JW revised the manuscript. All authors have read and approved the final manuscript.

\section{Acknowledgements}

Not applicable.

\section{References}

1. Feng, R.M., et al., Current cancer situation in China: good or bad news from the 2018 Global Cancer Statistics? Cancer Commun (Lond), 2019. 39(1): p. 22.

2. Valastyan, S. and R.A. Weinberg, Tumor metastasis: molecular insights and evolving paradigms. Cell, 2011. 147(2): p. 275-92.

3. Boccaletto, P., et al., MODOMICS: a database of RNA modification pathways. 2017 update. Nucleic Acids Res, 2018. 46(D1): p. D303-D307.

4. Trixl, L. and A. Lusser, The dynamic RNA modification 5-methylcytosine and its emerging role as an epitranscriptomic mark. Wiley Interdiscip Rev RNA, 2019. 10(1): p. e1510.

5. Frye, M. and S. Blanco, Post-transcriptional modifications in development and stem cells. Development, 2016. 143(21): p. 3871-3881.

6. Schaefer, M., et al., RNA methylation by Dnmt2 protects transfer RNAs against stress-induced cleavage. Genes Dev, 2010. 24(15): p. 1590-5.

7. Blanco, S., et al., Aberrant methylation of tRNAs links cellular stress to neuro-developmental disorders. EMBO J, 2014. 33(18): p. 2020-39.

8. Khan, M.A., et al., Mutation in NSUN2, which encodes an RNA methyltransferase, causes autosomalrecessive intellectual disability. Am J Hum Genet, 2012. 90(5): p. 856-63.

9. Martinez, F.J., et al., Whole exome sequencing identifies a splicing mutation in NSUN2 as a cause of a Dubowitz-like syndrome. J Med Genet, 2012. 49(6): p. 380-5.

10. Van Haute, L., et al., Deficient methylation and formylation of mt-tRNA(Met) wobble cytosine in a patient carrying mutations in NSUN3. Nat Commun, 2016. 7: p. 12039.

11. Nakano, S., et al., NSUN3 methylase initiates 5-formylcytidine biogenesis in human mitochondrial tRNA(Met). Nat Chem Biol, 2016. 12(7): p. 546-51.

12. Yi, J., et al., Overexpression of NSUN2 by DNA hypomethylation is associated with metastatic progression in human breast cancer. Oncotarget, 2017. 8(13): p. 20751-20765.

13. Gao, Y., et al., NOP2/Sun RNA methyltransferase 2 promotes tumor progression via its interacting partner RPL6 in gallbladder carcinoma. Cancer Sci, 2019. 110(11): p. 3510-3519.

14. Lu, L., et al., High tRNA Transferase NSUN2 Gene Expression is Associated with Poor Prognosis in Head and Neck Squamous Carcinoma. Cancer Invest, 2018. 36(4): p. 246-253. 
15. Frye, M. and F.M. Watt, The RNA methyltransferase Misu (NSun2) mediates Myc-induced proliferation and is upregulated in tumors. Curr Biol, 2006. 16(10): p. 971-81.

16. Okamoto, M., et al., Frequent increased gene copy number and high protein expression of tRNA (cytosine-5-)-methyltransferase (NSUN2) in human cancers. DNA Cell Biol, 2012. 31(5): p. 660-71.

17. Chen, R.X., et al., N(6)-methyladenosine modification of circNSUN2 facilitates cytoplasmic export and stabilizes HMGA2 to promote colorectal liver metastasis. Nat Commun, 2019. 10(1): p. 4695.

18. Goodarzi, H., et al., Modulated Expression of Specific tRNAs Drives Gene Expression and Cancer Progression. Cell, 2016. 165(6): p. 1416-1427.

19. Lind, D.S., Arginine and cancer. J Nutr, 2004. 134(10 Suppl): p. 2837S-2841S; discussion 2853 S.

20. Weiger, T.M. and A. Hermann, Cell proliferation, potassium channels, polyamines and their interactions: a mini review. Amino Acids, 2014. 46(3): p. 681-8.

21. Cole, C., et al., Filtering of deep sequencing data reveals the existence of abundant Dicer-dependent small RNAs derived from tRNAs. RNA, 2009. 15(12): p. 2147-60.

22. Lee, Y.S., et al., A novel class of small RNAs: tRNA-derived RNA fragments (tRFs). Genes Dev, 2009. 23(22): p. 2639-49.

23. Fu, H., et al., Stress induces tRNA cleavage by angiogenin in mammalian cells. FEBS Lett, 2009. 583(2): p. 437-42.

24. Luan, N., et al., TRF-20-MONK5Y93 suppresses the metastasis of colon cancer cells by impairing the epithelial-to-mesenchymal transition through targeting Claudin-1. Am J Transl Res, 2021. 13(1): p. 124-142.

25. Luan, N., et al., Dicer1 Promotes Colon Cancer Cell Invasion and Migration Through Modulation of tRF-20-MEJB5Y13 Expression Under Hypoxia. Front Genet, 2021. 12: p. 638244.

26. Shen, Y., et al., Transfer RNA-derived fragments and tRNA halves: biogenesis, biological functions and their roles in diseases. J Mol Med (Berl), 2018. 96(11): p. 1167-1176.

27. Shi, L., et al., Estrogen receptor (ER) was regulated by RNPC1 stabilizing mRNA in ER positive breast cancer. Oncotarget, 2015. 6(14): p. 12264-78.

28. Cai, X., et al., HBXIP-elevated methyltransferase METTL3 promotes the progression of breast cancer via inhibiting tumor suppressor let-7g. Cancer Lett, 2018. 415: p. 11-19.

29. Li, S., Z. Xu, and J. Sheng, tRNA-Derived Small RNA: A Novel Regulatory Small Non-Coding RNA. Genes (Basel), 2018. 9(5).

30. Reuter, S., et al., Oxidative stress, inflammation, and cancer: how are they linked? Free Radic Biol Med, 2010. 49(11): p. 1603-16.

31. Soares, A.R. and M. Santos, Discovery and function of transfer RNA-derived fragments and their role in disease. Wiley Interdiscip Rev RNA, 2017. 8(5).

32. Bohnsack, K.E., C. Hobartner, and M.T. Bohnsack, Eukaryotic 5-methylcytosine (m(5)C) RNA Methyltransferases: Mechanisms, Cellular Functions, and Links to Disease. Genes (Basel), 2019. 10(2). 
33. Squires, J.E., et al., Widespread occurrence of 5-methylcytosine in human coding and non-coding RNA. Nucleic Acids Res, 2012. 40(11): p. 5023-33.

34. Brzezicha, B., et al., Identification of human tRNA:m5C methyltransferase catalysing introndependent $m 5 \mathrm{C}$ formation in the first position of the anticodon of the pre-tRNA Leu (CAA). Nucleic Acids Res, 2006. 34(20): p. 6034-43.

35. Auxilien, S., et al., The human tRNA m (5) C methyltransferase Misu is multisite-specific. RNA Biol, 2012. 9(11): p. 1331-8.

36. Delaunay, S. and M. Frye, RNA modifications regulating cell fate in cancer. Nat Cell Biol, 2019. 21(5): p. 552-559.

37. Barbieri, I. and T. Kouzarides, Role of RNA modifications in cancer. Nat Rev Cancer, 2020. 20(6): p. 303-322.

38. Blanco, S., et al., Stem cell function and stress response are controlled by protein synthesis. Nature, 2016. 534(7607): p. 335-40.

\section{Figures}




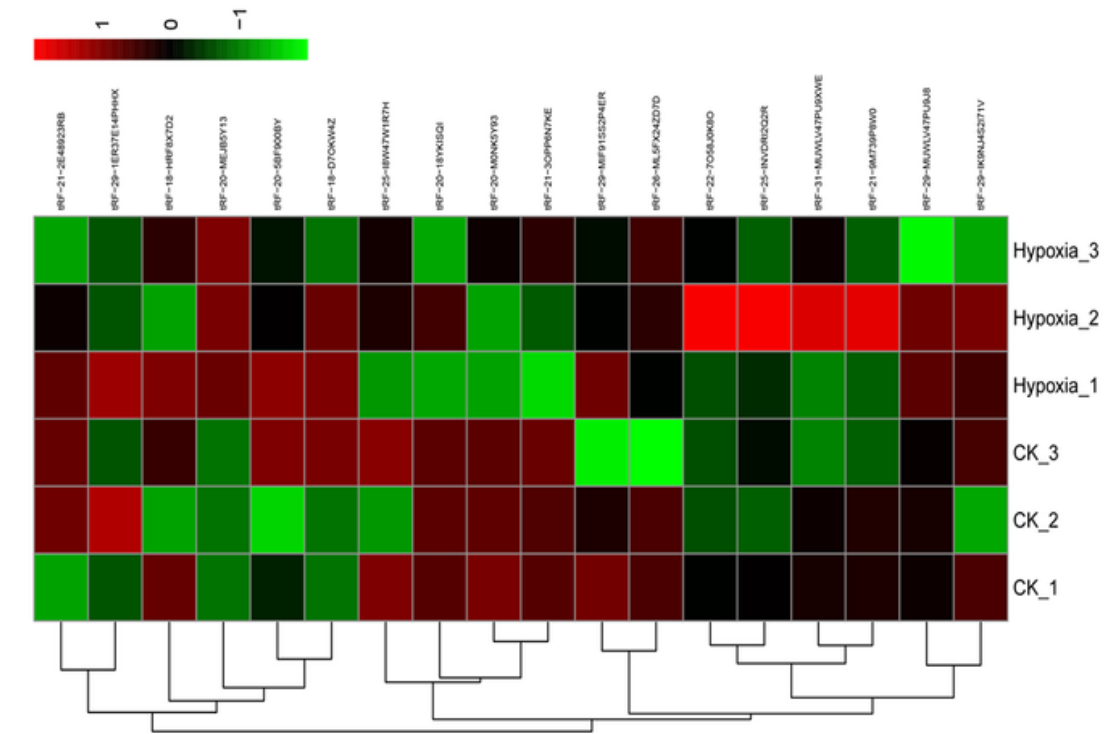

b

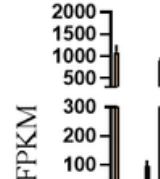

$\square$ Control

$\square$ Hypoxia

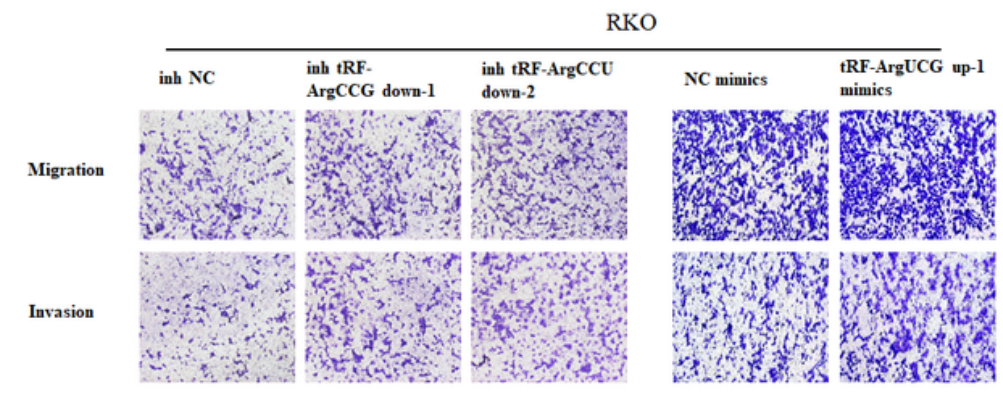

SW480
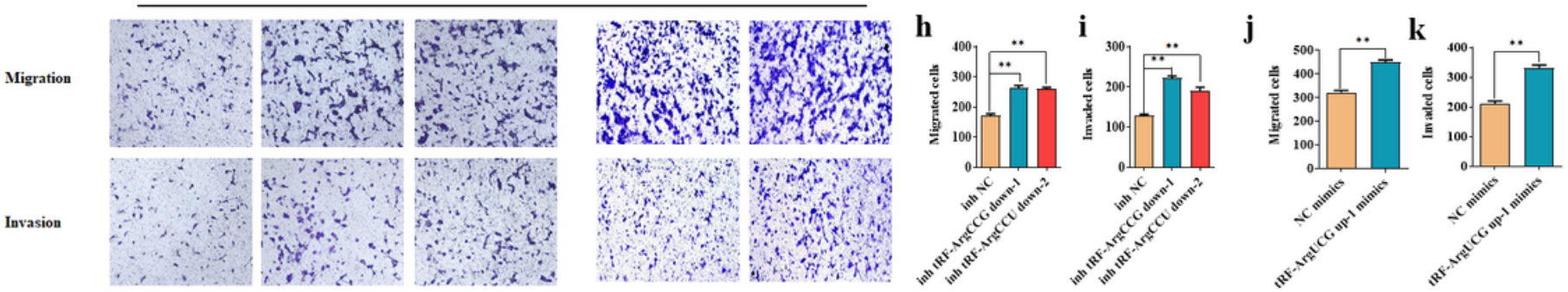

\section{Figure 1}

Separation of significantly changed tRFs and their roles on colon cancer metastasis. a Heat maps showing the top differentially expressed tRFs in colon cancer under hypoxia compared to normal oxygen. The red shades and blue shades represent high expression and low expression, respectively. b Significantly discrepant expressed tRFs were found by high-throughput sequencing analysis. C RT-PCR analysis was used to verify the expression of 18 kinds of tRFs in cell lines. Actin was the internal control. $\mathrm{d}, \mathrm{e}, \mathrm{h}, \mathrm{i}$ Transwell migration and invasion assays were performed to detect the motility of RKO and SW480 cells transfected with tR down- 1 inhibitor, tRF-ArgCCU down- 2 inhibitor. Columns are the average 
of three independent experiments. $f, g, j, k$ tRF-ArgUCG up-1 overexpression led to increased migration and invasion of RKO and SW480 cells.

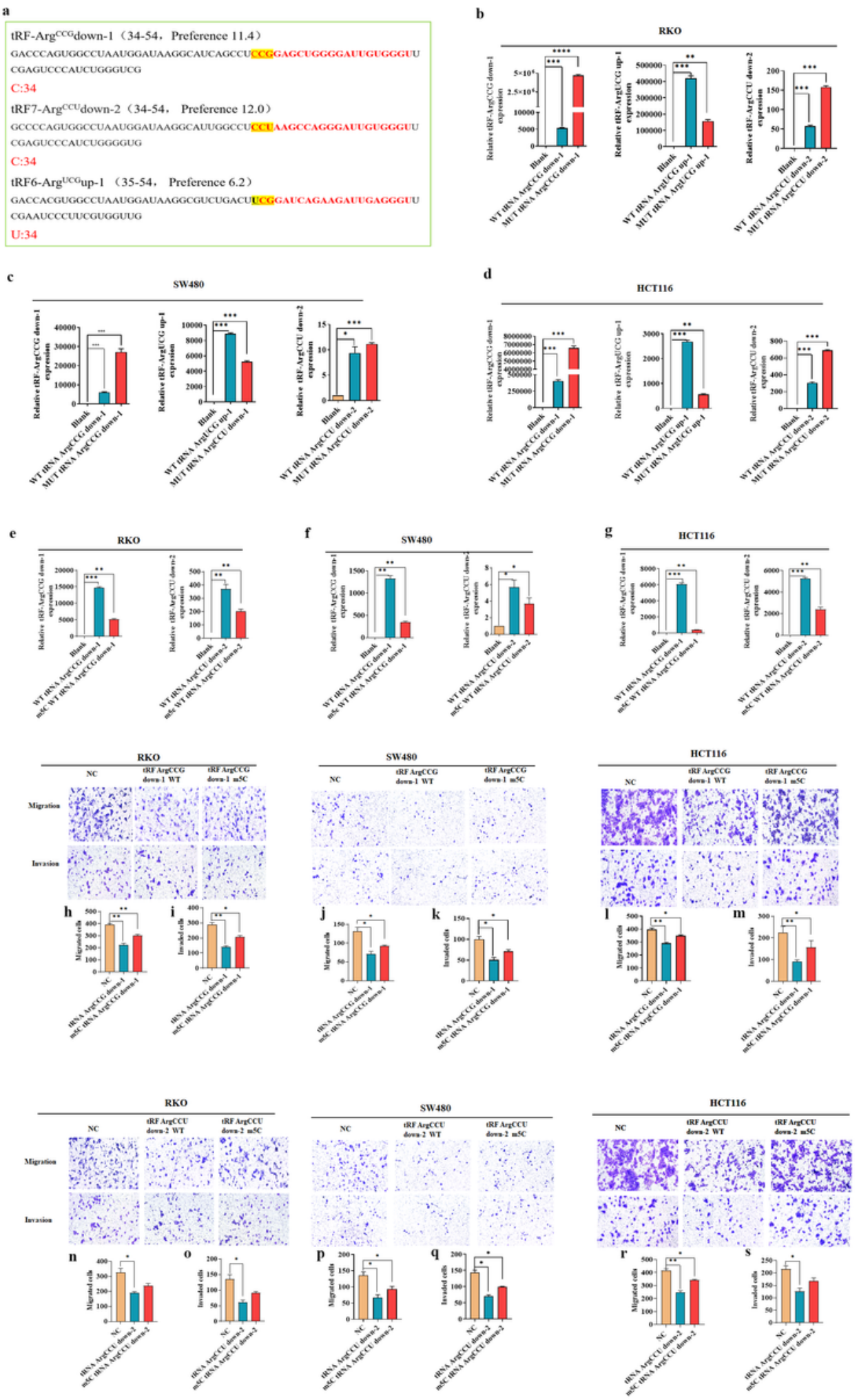

Figure 2

Key point of tRNA Arg plays a key role in tRNA leavage and tRF-mediated metastasis. a The location of the differential tRF on the corresponding tRNA and the position 34 base. The underlined is the antisense codon, and the red mark is the position of tRF. b, c, d qPCR assays identify the tRF-ArgCCG down-1, tRF- 
ArgUCG up-1 and tRF-ArgCCU down-2 expression of RKO, SW480 and HCT116 cells transfected with wildtype and mutant tRNA-5/6/7 24h. e, $\mathrm{f}, \mathrm{g}$ qPCR to examine the expression level of tRF ArgCCG down-1 and tRF-ArgCCU down-2 in RKO/SW480/HCT116 cells transfected with WT and added methylated m5C tRNA ArgCCU down-1 and tRNA ArgCCU down-2. h-s compared to NC groups, tRNA ArgCCG down-1 and tRFArgCCU down-2 groups could remarkably inhibit colon cancer cells invasion and migration. However, when compared to that of $\mathrm{C} 34$ without m5C methylation, tRF-ArgCCG down-1 and tRF-ArgCCU down-2 produced from C34 methylated tRNA ArgCCG down-1 and tRNA ArgCCU down-2 weakened the ability of colon cancer cells invasion and migration.

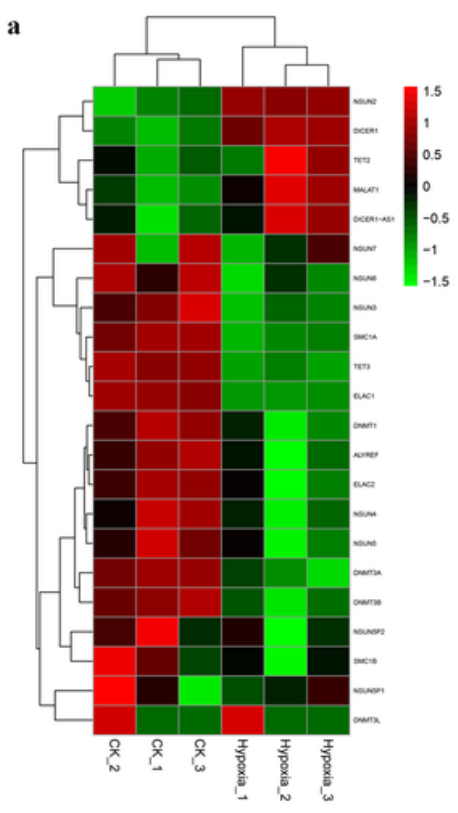

b
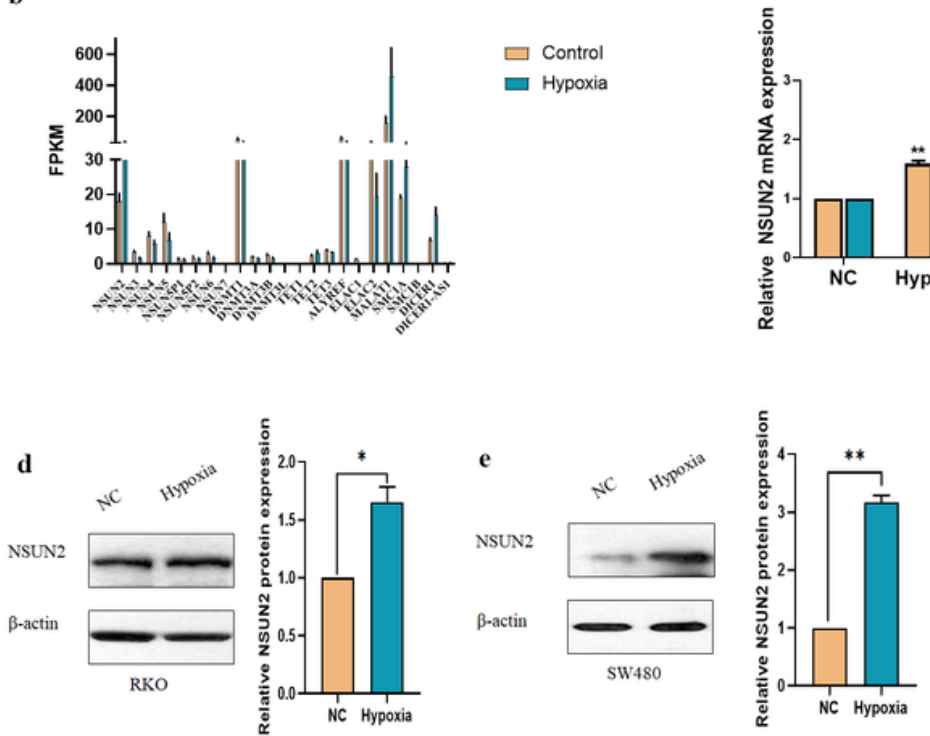

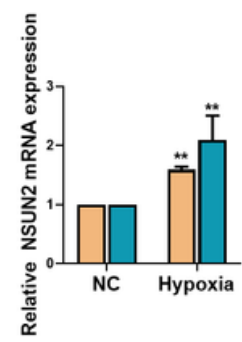

ㅁ RKO
$5 W 480$

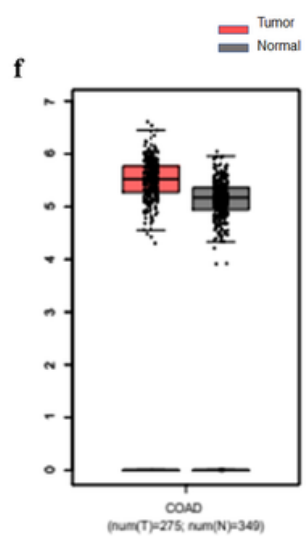

g

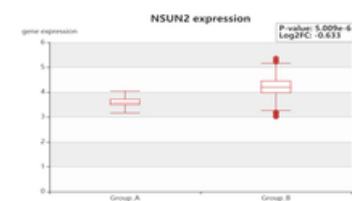

$\pm$

i

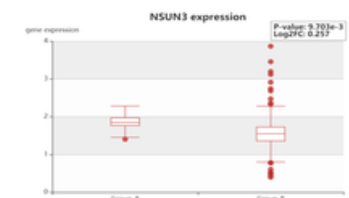

k

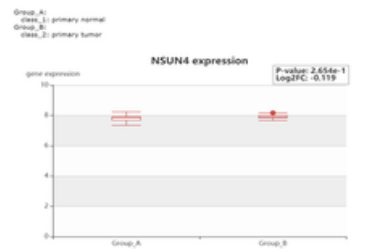

$=$ h
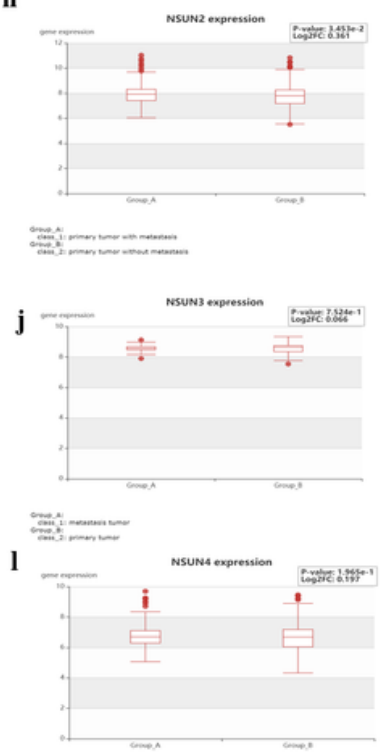

$=$ m
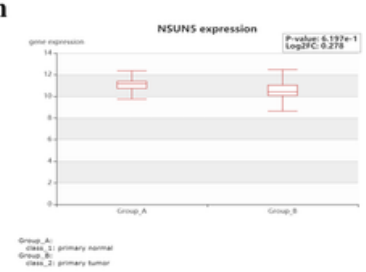

o
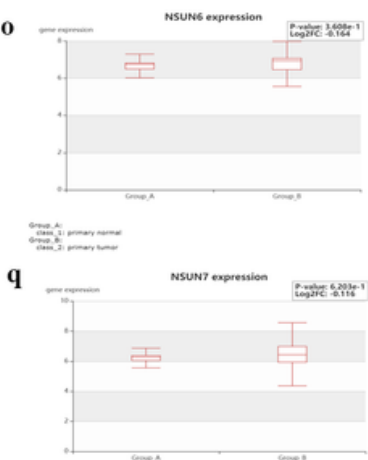

$\pm$ n

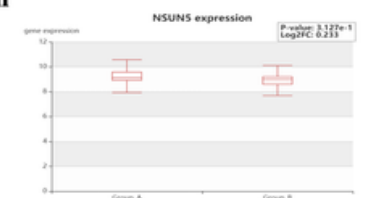

$=1$
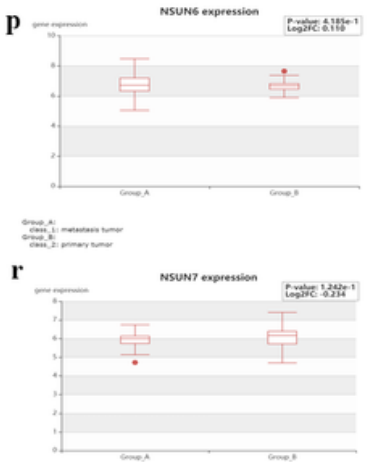

$\equiv-$

Figure 3 
NSUN2 overexpression characterized colon cancer with predictive values. a Heatmap for differentially expressed m5C RNA methylated enzymes and RNase in colon cancer tissues under hypoxia compared with that in normoxia conditions. b Further analysis of heatmap. c Relative RNA expression of NSUN2 in RKO and SW480 cells under hypoxia analyzed by RT-qPCR. d, e Relative protein expression of NSUN2 in RKO and SW480 cells under hypoxia analyzed by western blot. $\mathrm{f}, \mathrm{g}$, h NSUN2 upregulation is associated with tumour in primary focus as well as advanced progression in colon cancer. i-r other NSUN methyltransferases (such as NSUN 3-7) expressed no significantly difference between normal tissue and primary tumour focus, nor primary tumour focus and metastasis focus.
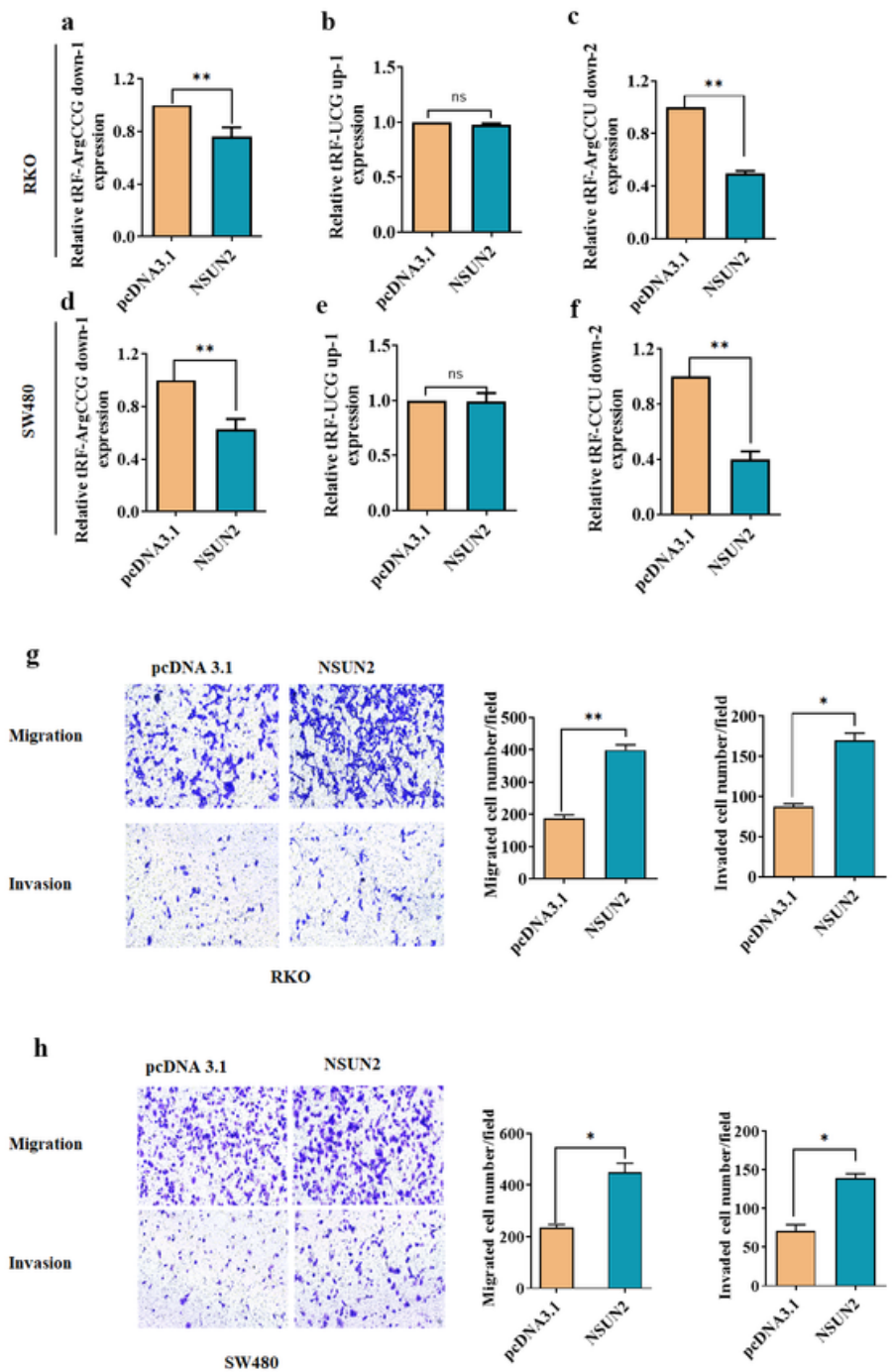
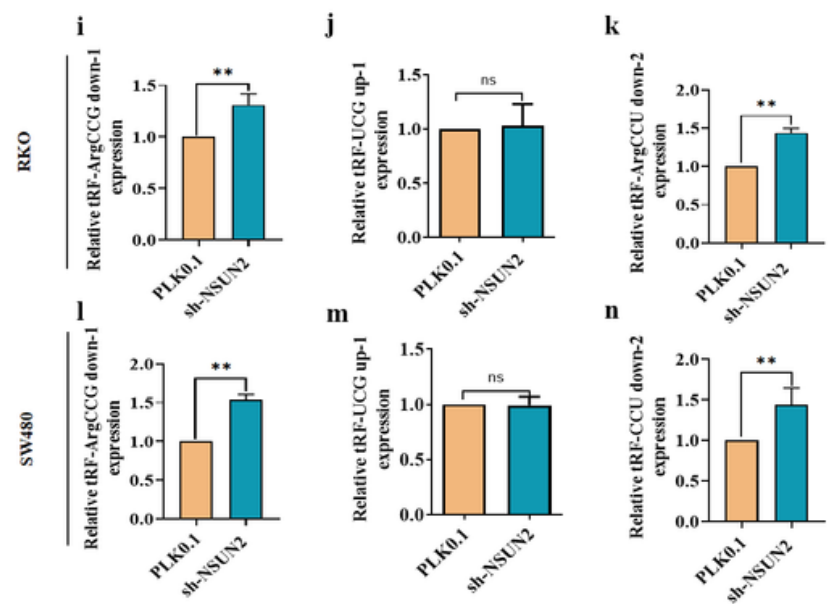

o
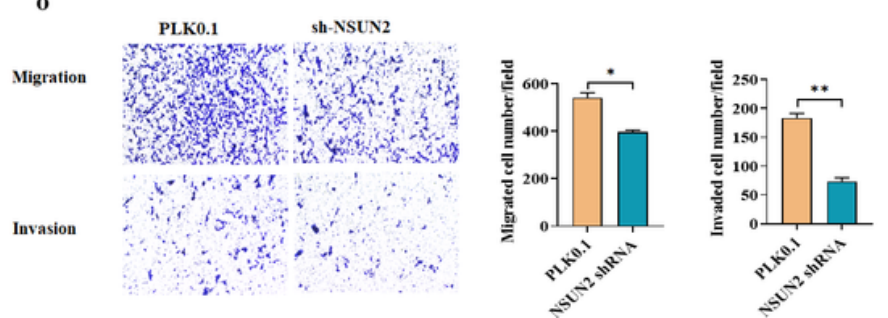

RKo
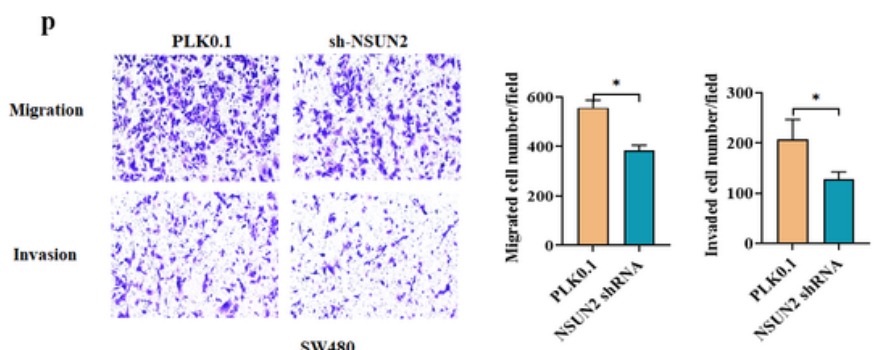

\section{Figure 4}

NSUN2 played a role as a promoter of colon cancer. a-f PCR assays results showed that after NSUN2 knockdown, the expression of tRNA ArgCCG down-1 and tRF-ArgCCU down-2 increased, while the expression of tRF ArgUCG up-1 remained basically unchanged. $\mathrm{g}$ Transwell assays analysis showed that down-regulation of NSUN2 could decrease the invasion and migration ability. i-n After NSUN2 overexpression, the expression of tRNA ArgCCG down-1 and tRF-ArgCCU down-2 decreased, while the 
expression of tRF ArgUCG up-1 remained basically unchanged. $g, h, 0, p$ overexpression and downregulated expression of NSUN2 could regulate the invasion and migration ability of colon cancer cells.
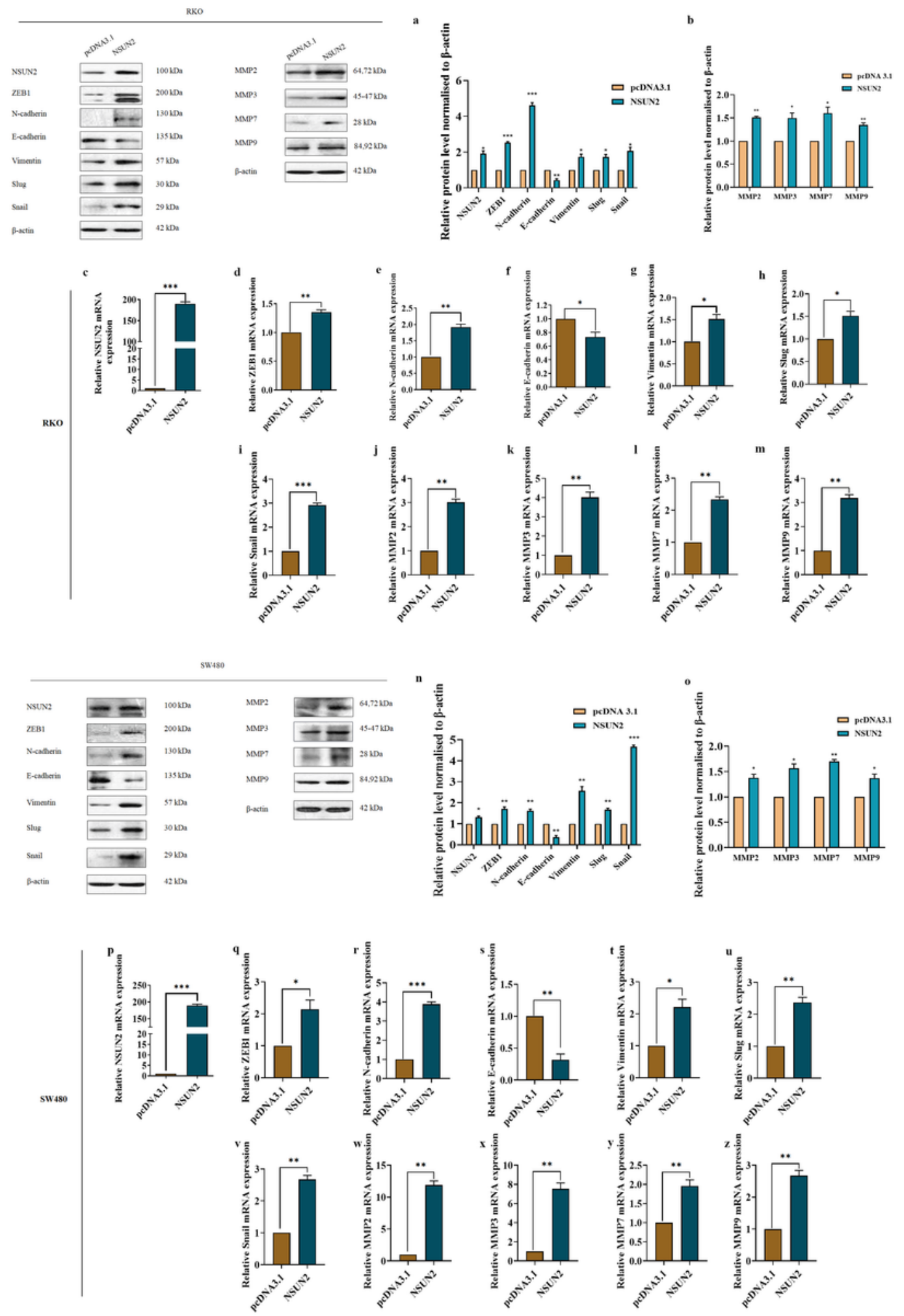

Figure 5

a, b, n, o EMT-related proteins were detected by western blot in RKO and SW480 cells after overexpression of NSUN2, and the analysis showed that N-cadherin, and Vimentin, ZEB1, Slug, Snail, MMP2, MMP3, 
MMP7, MMP9 increased, while E-cadherin decreased. c-m, p-z the changed mRNA expression of EMTrelated markers with NSUN2 overexpression in RKO and SW480 cells examined by qPCR assays.

a

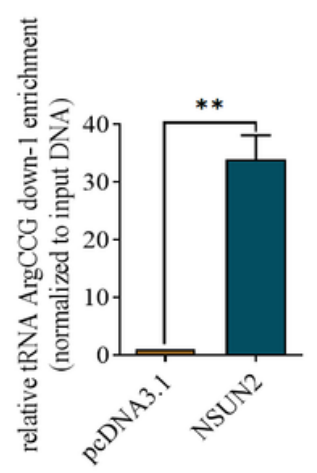

b

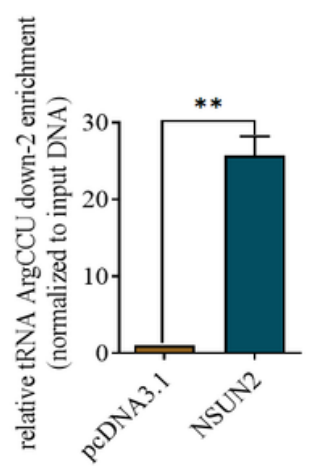

c

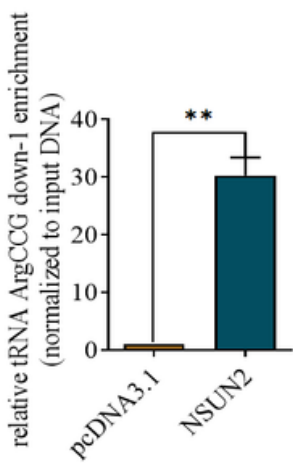

d

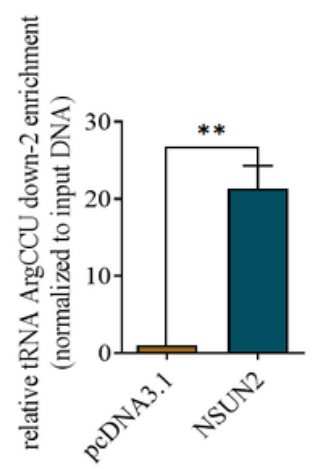

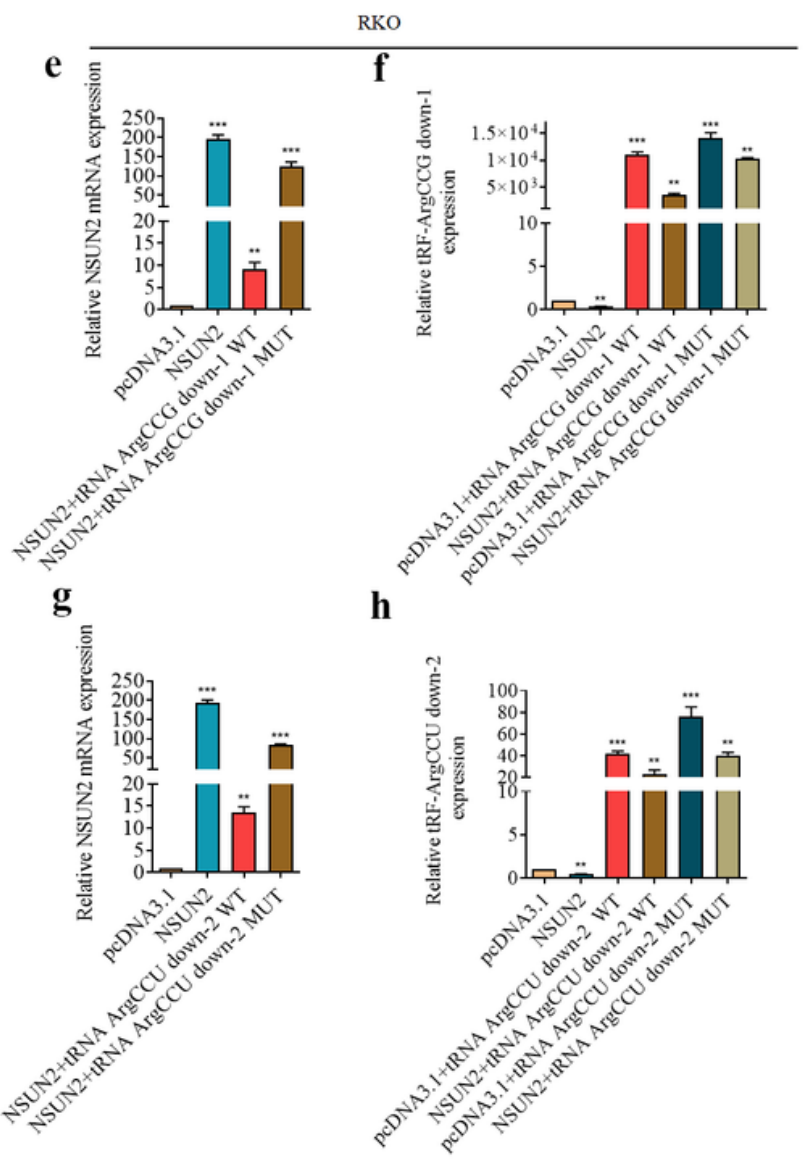

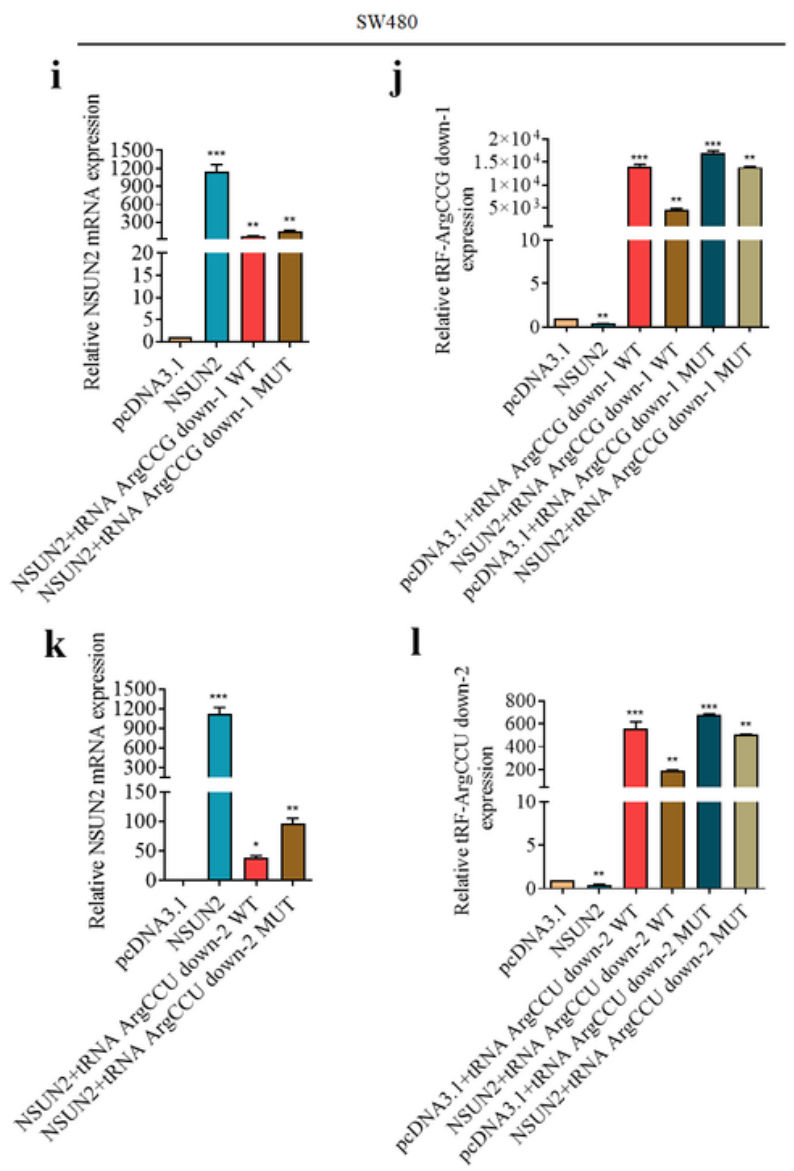

\section{Figure 6}

NSUN2 and methylation of tRNA Arg C34 together determine tRNA Arg cleavage. $a, b$ RIP and PCR analysis of RKO cells identified the binding of NSUN2 and tRNA Arg C34 in the tRNA ArgCCG down-1, tRFArgCCU down-2. The input was used as an internal positive control. When compared to the input group, NSUN2 overexpression markedly increased the binding of NSUN2 and tRNA ArgCCU down-1, tRNA ArgCCU down-2. c, d RIP and PCR analysis of RKO cells identified the binding of m5C antibodies and tRNA Arg C34 in the tRNA ArgCCG down-1, tRF-ArgCCU down-2. The input was used as an internal 
positive control. When compared to the input group, NSUN2 overexpression markedly increased the binding of m5C antibodies and tRNA ArgCCU down-1, tRNA ArgCCU down-2. e, g, i, k tRNA Arg CCG down1 and tRNA ArgCCU down-2 without C34 mutations markedly suppressed NSUN2 expression in RKO and SW480 cells, compared to those without C34 mutations. $\mathrm{f}, \mathrm{h}, \mathrm{j}, \mathrm{I}$ The expression of tRF-Arg CCG down-1 and tRF-ArgCCU down-2 with C34 mutations markedly down-regulated than without C34 mutations after NSUN2 overexpression. Within the group of tRNA ArgCCG down-1, tRNA ArgCCU down-2 without C34 mutations, overexpression of NSUN2 could lead to the downregulation of tRF-Arg CCG down-1 and tRFArgCCU down-2. Within the group of tRNA ArgCCG down-1, tRNA ArgCCU down-2 with C34 mutations, NSUN2 overexpression hardly play a role in depressing the expression of tRNA ArgCCG down-1, tRFArgCCU down-2. 

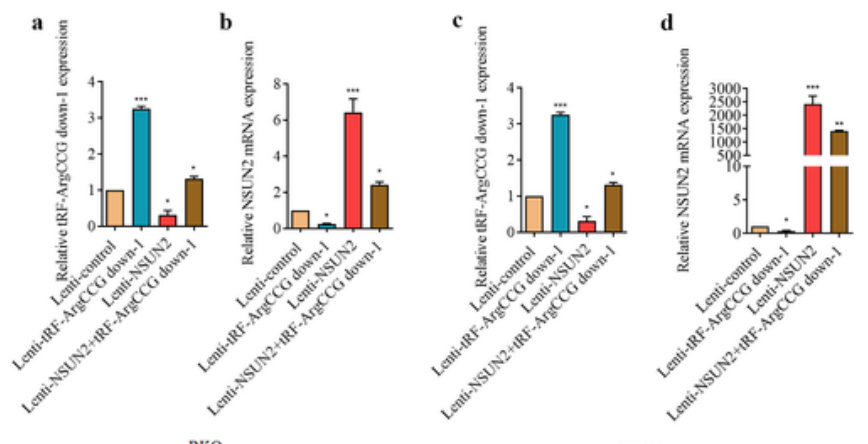

RKo

sw480
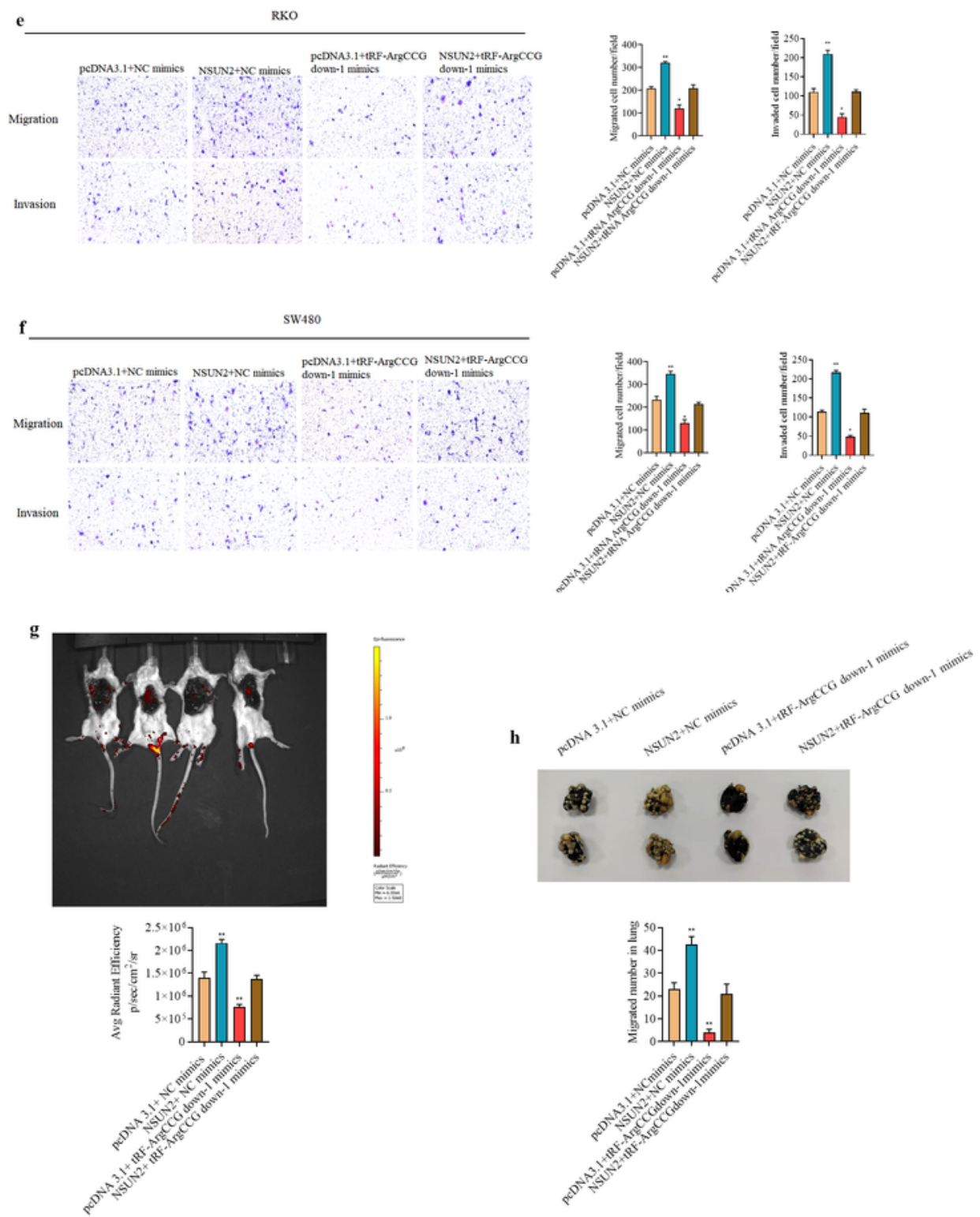

\section{Figure 7}

NSUN2 promotes colon cancer metastasis partilally via tRNA ArgCCG down-1 in vitro and in vivo. a, c Overexpression of tRNA ArgCCG down-1 can partially eliminate the effect of NSUN2 on suppressing tRNA ArgCCG down-1 expression. b, d tRF-ArgCCG down-1 produced from tRNA ArgCCG down-1 has a negative feedback effect on the expression of NSUN2. e, $f$ The interaction of NSUN2 and tRNA ArgCCG down-1 could weaken NSUN2 promoting metastasis ability and damage tRF-ArgCCG down-1 inhibiting 
metastasis ability by reducing its production. $\mathrm{g}$. The BALBC white mice implanted with NSUN2+/ NC mimics CT-26 cells exhibited significantly higher GFP activity in lung, while the GFP signal of mice bearing pcDNA 3.1/ tRNA ArgCCG down-1 mimics RKO cells were much lower than that of mice in the NC group. Significantly, the GFP signal of mice in the NSUN2+/ tRF-Arg CCG down-1 mimics group was similar to the NC group. $\mathrm{h}$ General view lung tissue of each mice.

\section{Supplementary Files}

This is a list of supplementary files associated with this preprint. Click to download.

- FigS1.tif

- Figs2.tif

- FigureS3..tif

- SupplementaryTables1..docx

- SupplementaryTableS2..docx 